10IKC-176

\title{
GEOCHEMISTRY AND MICROSTRUCTURE OF DIAMONDITES
}

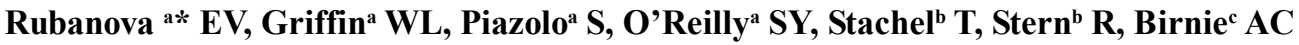 \\ "ARC Centre of Excellence for Core to Crust Fluid Systems and GEMOC ARC National Key Centre, \\ Department of Earth and Planetary Sciences, Macquarie University, NSW, 2109, Australia \\ ${ }^{b}$ Department of Earth and Atmospheric Sciences, University of Alberta, Canada, T6G 2E3 \\ ${ }^{c}$ Kopane Diamonds Plc, 235-237 Vauxhall Bridge Road, London SWIV IEJ \\ ekaterina.rubanova@gmail.com
}

Diamondites are rocks consisting dominantly of polycrystalline diamonds; cavities may be lined with silicate minerals and transparent euhedral diamonds. Previously they have been interpreted as primary precipitates, formed by rapid crystallization from a fluid. However, these data have been obtained from unpolished surfaces or from disaggregated material, and thus lack microstructural context. For this study polished plates of diamondites were prepared for detailed observation of internal structure and in-situ analysis of trace elements. To understand the nature of diamond-forming processes we have studied African diamondites, polycrystalline diamonds and diamondiferous eclogites from Lesotho. In the eclogites, diamondites occur enclosed in large garnet grains, while monocrystalline diamonds and sulfides are related to cpx-bearing metasomatic veins that crosscut the garnets.

The internal structure of the diamondites, as revealed by $\mathrm{CL}$ and Electron Backscattered Diffraction (EBSD), shows that diamondites underwent plastic deformation and recrystallisation under mantle conditions. Plastic deformation induced the generation of dislocations, their migration to form subgrain boundaries, activation of several slip systems, grain-size reduction by subgrain rotation, nucleation and growth, and migration and recovery of grain boundaries. The progressive recrystallisation was accompanied by significant rearrangement of both dislocations and chemical components, so that in highly deformed samples, silicates mostly occur interstitially. Interstitial silicates may have undergone interaction with metasomatic fluids penetrating along diamond grain boundaries. They have higher mg\# $(0.78)$ than original unaltered inclusions ( 0.70 to 0.53$)$. Coexisting clinopyroxene and garnet inclusions from the least deformed sample give a temperature of $1200^{\circ} \mathrm{C}$.

The $\delta^{13} \mathrm{C}$ of the studied diamondites ranges between $-16.2 \%$ and $-29.4 \%$ (VPDB, precision $0.1 \%$ ) with almost no intra-sample variation. Cisotope analysis by ion microprobe shows that the octahedral crystals from Northern Lesotho eclogites have carbon-isotope compositions similar to the diamondites. A diamond overgrowth in one of the samples has higher $\delta^{13} \mathrm{C}(-19.4$ to $19.9 \%$ ) than the internal part of the diamond $(23.6$ to $-24.8 \%$ ). Similar zoning (outward ${ }^{13} \mathrm{C}$ enrichment) is observed in diamondite from another eclogite sample. A transparent diamond from another eclogite is zoned from $-22.0 \%$ in the core, to $-6.3 \%$ at an intermediate growth stage, and to $-11.8 \%$ toward the edge of the diamond, implying growth from isotopically distinct fluids. The similarity in $\delta^{13} \mathrm{C}$ of diamondites and transparent crystals indicates both formed in the same crystallisation system but at different levels of carbon saturation. Variations in the composition 


\section{0 $^{\text {th }}$ International Kimberlite Conference, Bangalore - 2012}

of mantle fluids may be responsible for changes in crystal habit, the composition of interstitial silicates and the carbon-isotope composition of diamonds. LAM-ICPMS analyses of the clearest parts of diamondites suggest that the fluids involved in their precipitation were in the kimberlite-carbonatite spectrum.

This study shows that polycrystalline diamond aggregates (diamondites) should not be generally interpreted as products of primary crystallisation shortly before eruption. Internal structure plays an important role in the interpretation of their genesis. We suggest that deformation and recrystallization of diamondites may be related to fluid/melt infiltration (metasomatic processes), which also changed the crystallisation environment and provided the carbon for the formation of octahedral crystals in metasomatic veins. 